\section{Assessment of dehydrothermally} cross-linked collagen membrane for guided bone regeneration around peri-implant dehiscence defects: a randomized single-blinded clinical trial

\author{
Jae-Hong Lee ${ }^{1,+}$, Jung-Seok Lee ${ }^{1,+}$, Won-Sun Baek ${ }^{1}$, Hyun-Chang Lim², \\ Jae-Kook Cha', Seong-Ho Choi ${ }^{1}$, Ui-Won Jung ${ }^{1, *}$ \\ ${ }^{1}$ Department of Periodontology, Research Institute for Periodontal Regeneration, Yonsei University College \\ of Dentistry, Seoul, Korea \\ ${ }^{2}$ Department of Periodontology, Kyung Hee University School of Dentistry, Seoul, Korea
}

Purpose: The aim of this study was to determine the clinical feasibility of using dehydrothermally cross-linked collagen membrane (DCM) for bone regeneration around peri-implant dehiscence defects, and compare it with non-cross-linked native collagen membrane (NCM).

Methods: Dehiscence defects were investigated in twenty-eight patients. Defect width and height were measured by periodontal probe immediately following implant placement (baseline) and 16 weeks afterward. Membrane manipulation and maintenance were clinically assessed by means of the visual analogue scale score at baseline. Changes in horizontal thickness at $1 \mathrm{~mm}, 2 \mathrm{~mm}$, and $3 \mathrm{~mm}$ below the top of the implant platform and the average bone density were assessed by cone-beam computed tomography at 16 weeks. Degradation of membrane was histologically observed in the soft tissue around the implant prior to re-entry surgery.

Results: Five defect sites (two sites in the NCM group and three sites in the DCM group) showed soft-tissue dehiscence defects and membrane exposure during the early healing period, but there were no symptoms or signs of severe complications during the experimental postoperative period. Significant clinical and radiological improvements were found in all parameters with both types of collagen membrane. Partially resorbed membrane leaflets were only observed histologically in the DCM group.

Conclusions: These findings suggest that, compared with NCM, DCM has a similar clinical expediency and possesses more stable maintenance properties. Therefore, it could be used effectively in guided bone regeneration around dehiscence-type defects.

Keywords: Augment bone graft, Controlled clinical trial, Cross-linking, Membrane.

\section{INTRODUCTION}

Guided bone regeneration (GBR) has become an indispensable technique for enhancing new bone formation around peri-implant dehiscence defects [1,2]. Non-cross-linked native collagen membrane (NCM) is the most widely used type of membrane in the GBR technique. It shows excellent biocompatibility with a low immune response, and has the advantage of promoting wound healing by inducing fibroblast migration [3-5]. In addition, the bone regeneration capacity of NCM is similar to that of nonresorbable membrane [6]. Despite these advantages, the shortened functional period due to a loss of cell occlusiveness and greater susceptibility to degradation by periodontal bacteria may restrict bone regeneration when using NCM $[7,8]$.

Various cross-linking methods have been investigated and developed with the aim of

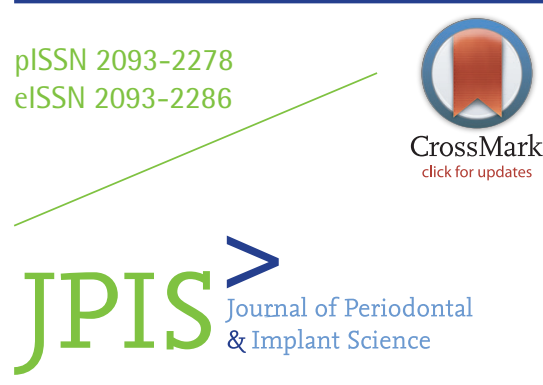

\section{Research Article}

J Periodontal Implant Sci 2015;45:229-237 http://dx.doi.org/10.5051/jpis.2015.45.6.229

Received: Nov. 4, 2015

Accepted: Dec. 2, 2015

*Correspondence:

Ui-Won Jung

Department of Periodontology, Yonsei University

College of Dentistry, 50-1 Yonsei-ro,

Seodaemun-gu, Seoul 03722, Korea

E-mail: drjew@yuhs.ac

Tel: +82-2-2228-3185

Fax: +82-2-392-0398

${ }^{\dagger}$ Jae-Hong Lee and Jung-Seok Lee contributed equally to this study.

This is an Open Access article distributed under the terms of the Creative Commons Attribution Non-Commercia License (http://creativecommons.org/licenses/by-nc/3.0/). 
improving the mechanical properties and compensating for the disadvantages of NCM [9]. Chemical and physical modifications are important for increasing the degree of cross-linking between collagen fibers, which varies significantly with the cross-linking method used [10]. However, all cross-linked membranes reportedly increase thermal stability, mechanical strength, and resistance to enzyme activity [11].

Chemically cross-linked collagen membrane reportedly exhibits biocompatibility with a minimal inflammatory response and has been used for GBR; however, several randomized controlled clinical studies have also demonstrated that it exerts significant adverse effects on bone regeneration [12-15]. For example, Becker et al. [14] reported that while chemically cross-linked collagen membrane provides predictable bone augmentation, it is also frequently associated with premature membrane exposure and wound infections. In addition, Annen et al. [15] showed that chemically crosslinked collagen membrane with an extended resorption time was associated with significantly more complications and a lower bone regeneration efficacy compared to NCM.

Dehydrothermal (DHT) treatment is a major physical modification method used in the production of a cross-linking collagen matrix. In vitro studies have demonstrated that DHT cross-linked collagen membrane (DCM) exhibits a high tensile strength and resistance to degradation, and reduced cytotoxic responses [16,17]. Rothamel et al. [18] showed that DCM and NCM have similar biocompatibility in rabbits, and that DCM provides an adequate environment for bone remodeling with sufficient vascularization during the initial healing phase as well as long-term structural stability. However, despite these positive results in animal studies, few clinical and controlled human studies have assessed the efficacy and safety of DCM.

The aim of this study was to determine the clinical feasibility of DCM for use in bone regeneration around peri-implant dehiscence defects, and compare it with that of NCM.

\section{MATERIALS AND METHODS}

\section{Study design}

A randomized, single-blinded, single-center clinical trial was conducted to evaluate the clinical feasibility and bone regeneration capacity around peri-implant dehiscence defects of two types of membrane. The study was approved by the Institutional Review Board (IRB) for Clinical Research at Dental Hospital of Yonsei University (approval no. 2-2013-0021). All patients provided written fully informed consent in accordance with IRB guidelines for enrollment, and the study was conducted in accordance with the Declaration of Helsinki and the Guidelines on Good Clinical Practice [19].

\section{Study population}

A total of 43 patients who needed single-tooth implant treatment from August 2013 to October 2014 were included in this clinical study. The following inclusion criteria were applied: (1) male or female aged $\geq 20$ years, (2) healthy systemic condition (including well-controlled medical illnesses), (3) a vertical dehiscence defect (only on the buccal side) of $\geq 3 \mathrm{~mm}$ present immediately after implant placement, (4) secure primary stability of the implant, and (5) no allergic reaction to collagen. The following exclusion criteria were applied: (1) severe or uncontrolled systemic disease, (2) advanced or untreated periodontitis, (3) pregnancy or breastfeeding, (4) history of radiation therapy to the head or neck, (5) hormones or bisphosphonate therapy affecting bone or connective tissue metabolism, and (6) heavy smoking (>20 cigarettes/day).

\section{Sample size calculation}

The required sample size was determined using the two-sided $t$ test at an alpha level of 0.05 and a statistical power of $80 \%$. The threshold for differences in bone regeneration capacity between the NCM and DCM groups was set to $1.0 \mathrm{~mm}$, and the standard deviation was assumed to be the same for both groups according to the results of a previous study $[20,21]$. These parameters resulted in a required sample size of 28 patients, and so 30 patients were enrolled (15 patients in each group) to account for a potential dropout rate of $10 \%$. The statistical power was calculated using $\mathrm{G}^{*}$ Power 3.1 (University of Duesseldorf, Germany) [22].

\section{Randomization}

Randomization took place after implant placement using online databases for clinical trials (Sealed Envelope ${ }^{\mathrm{TM}}$, sealedenvelope. com). The 30 enrolled patients were assigned to either the NCM group $(n=15)$ or the DCM group $(n=15)$ according to computergenerated random numbers. None of the patients knew whether they received the control or experimental membrane until after the end of the study.

\section{Surgical procedures}

All steps in the surgical procedures and all evaluation parameters were calibrated in training and calibration sessions. All patients received antibiotics (amoxicillin $500 \mathrm{mg}$ or roxithromycin $150 \mathrm{mg}$ daily), a single dose of analgesic (ibuprofen 200mg), and mouthwash (GUM Activital, Sunstar, Osaka, Japan) after implant surgery for 7 days. Full-thickness flaps were elevated, with vertical incisions made when necessary. A surgical stent was prepared for the optimal implant position, and a sandblasted, large-grit,acid-etched (SLA) surface internal-type implant fixture was placed in accordance with the manufacturer's recommended protocol. A sealed randomization envelope was opened to allocate augmentation of the defect, with either porcine dermis-derived non-cross-linked type I and III collagen (BioGide ${ }^{\circledR}$ Geistlich Biomaterials, Wolhusen, Switzerland) or porcine pericardium-derived type I collagen membrane (OssGuide ${ }^{\circledR}$, Bioland, Cheongju, Korea) and xenograft bone substitutes (BioOss ${ }^{\circledR}$, Geistlich Biomaterials, Wolhusen, Switzerland, and CollaOss ${ }^{\circledR}$, Bioland, Cheongju, Korea). After implant placement in the ideal prosthetic position, the horizontal and vertical dehiscence defect was augmented. A collagen membrane was trimmed 
so that it extended $2-3 \mathrm{~mm}$ from the defect margin. Flaps were sutured with 6-0 absorbable sutures (Monosyn 6-0, B. Braun Aesculap, Tuttlingen, Germany), with a horizontal periosteal releasing incision used where necessary to attain primary and tension-free closure of the flap. Follow-up was performed three times during 8 weeks and additional care was arranged according to the needs of individual patients.

\section{Clinical analysis}

All horizontal and vertical defects were measured using a 15- $\mathrm{mm}$ UNC periodontal probe (CP 15 UNC, Hu-freidy, Chicago, IL, USA) at the time of implant installation and re-entry surgery. The same trained and calibrated examiners carried out all measurements. The following parameters were measured: (1) defect width (DW), measured as the linear distance between the widest points on the medial and distal sides of the buccal aspect; (2) defect height (DH), measured as the linear distance from the top of the implant platform to the initial bone-to-implant contact at the buccal aspect; (3) a change in the defect width $(\Delta \mathrm{DW})$, calculated as DW (re-entry surgery) - DW (baseline); and (4) a change in the defect height $(\Delta \mathrm{DH})$, calculated as $\mathrm{DH}$ (re-entry surgery) - DH (baseline).

The ease of manipulating and maintaining NCM and DCM in implant surgery was clinically assessed using scores on a visual analog scale (VAS) that ranged from 0 (very good) to 10 (very poor). The following parameters were measured: (1) manipulation of the membrane, degree of hydrophilicity and handling during the GBR procedure; and (2) maintenance of augmented bone substitutes, and whether covering the membrane after augmentation with bone materials improved the stability.

\section{Radiographic analysis}

Cone-beam computed tomography (CBCT; Alphard Vega, Asahi Roentgen, Kyoto, Japan) was performed to assess the horizontal thickness (HT) immediately after augmentation and at the time of re-entry surgery. The following parameters were measured (Figure 1): (1) horizontal thickness of horizontal augmented bone located 1, 2, and $3 \mathrm{~mm}$ below the top of the implant platform (HT1, HT2, and HT3); (2) changes in horizontal thickness at each level, calculated as HT (re-entry surgery) - HT (baseline) $\left(\Delta \mathrm{HT} 1, \Delta \mathrm{HT}_{2}\right.$, and $\left.\Delta \mathrm{HT} 3\right) ;(3)$ density of the newly formed bone located $1 \mathrm{~mm}$ below the top of the implant platform, assessed using the Hounsfield unit (HU) scale with computed-tomography image-processing software (OnDemand3D ${ }^{\circledR}$, CyberMed, Seoul, Korea) (bone density of HT1).

\section{Histological analysis}

After a healing period of 16 weeks, when there was sufficient keratinized tissue around the top of the implant cover screw, a thin strip-shaped soft-tissue biopsy sample was obtained prior to performing re-entry surgery and the connection of the abutment. The biopsy samples were fixed in 10\% neutral formalin for 10 days, and then trimmed and dehydrated in a graded series of alcohol solutions. All specimens were stained with hematoxylin-eosin and

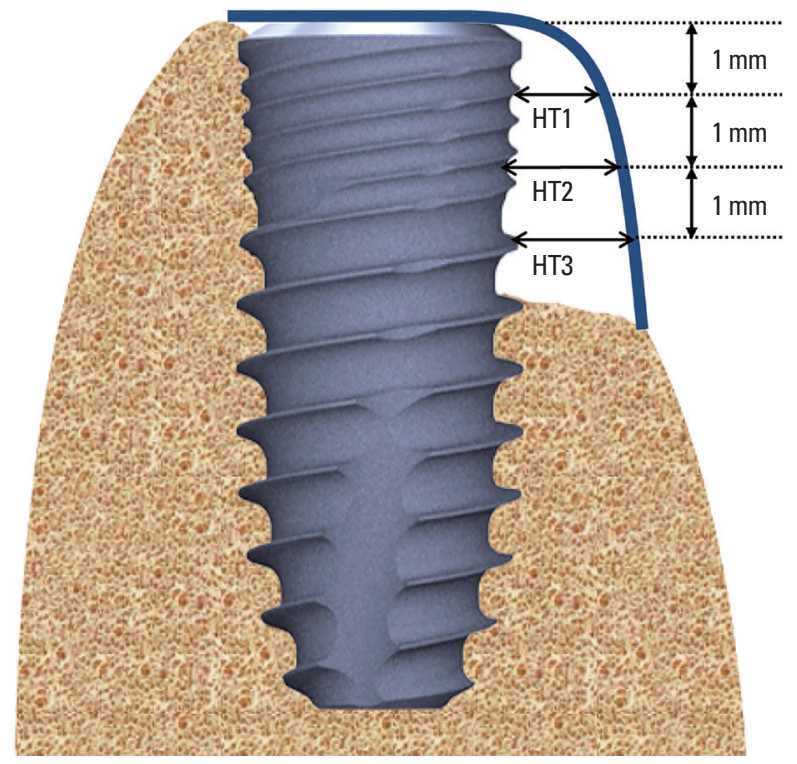

Figure 1. Mid-cross-sectional image of the implant placement site, showing measurement of horizontal thickness (HT) after augmentation and at the time of re-entry surgery. HT was calculated at three levels: 1, 2, and $3 \mathrm{~mm}$ below the top of the implant platform.

Masson's trichrome stains. The slides were observed under a light microscope (BX50, Olympus, Tokyo, Japan).

\section{Research protocol alteration}

During concurrent research that is currently still in progress, we determined that the use of different bone substitutes in the NCM and DCM groups interfered with evaluations of membrane efficiency. Therefore, after obtaining re-approval from the IRB, we used the same bone graft materials (BioOss ${ }^{\circledR}$ ) in both groups, and altered the study to focus on the clinical feasibility of the membrane.

\section{Statistical analysis}

The mean \pm standard deviation values and 95\% confidence intervals were estimated for each study group. Statistical analyses were performed with IBM SPSS Statistics (Version 21.0, IBM Corp, Armonk, NY, USA), using independent $t$-tests to compare the results between the NCM and DCM groups $(P<0.05)$.

\section{RESULTS}

\section{Patient enrollment}

The 30 enrolled patients who fulfilled the inclusion and exclusion criteria comprised 16 males and 14 females with a mean age of 53.3 years (range, 31 to 75 years). Two patients dropped out during the follow-up: one patient in the DCM group showed signs of local infection such as gingival swelling, redness, and pus discharge at the 4-week checkup, and so the implant fixture was removed; the other patient was in the NCM group and showed early exposure of the cover screw at the 8-week checkup, and so an 


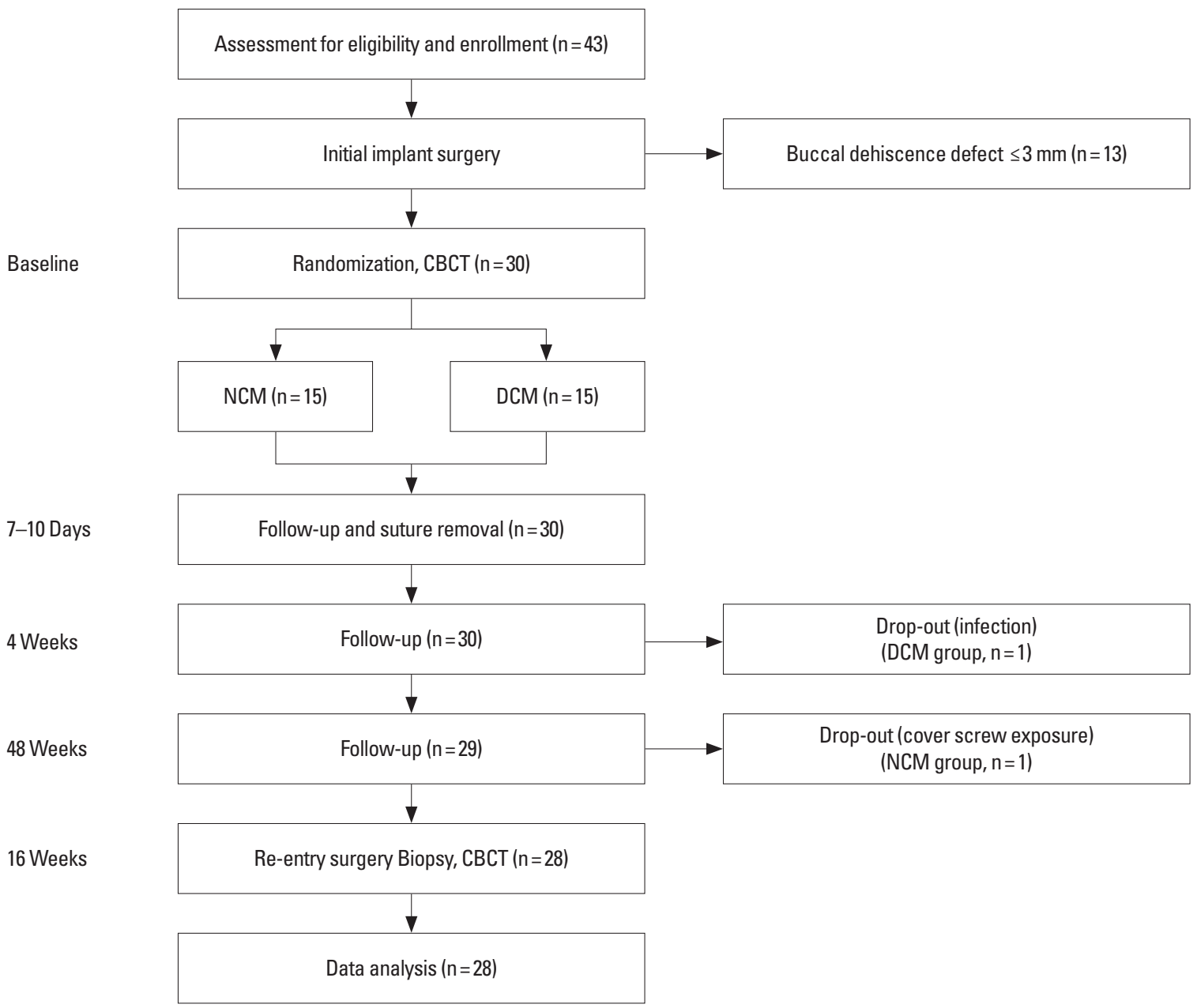

Figure 2. Flow chart of patient enrollment and reasons for exclusion. NCM: non-cross-linked native collagen membrane; DCM: dehydrothermally cross-linked collagen membrane; CBCT: cone-beam computed tomography.

Table 1. Characteristics of enrolled patients in the two study groups.

\begin{tabular}{|lccc|}
\hline Characteristic & NCM group & DCM group & Total \\
\hline Sample size & 14 & 14 & 28 \\
Gender & 9 & 5 & 14 \\
Male & 5 & 9 & 14 \\
Female & & & \\
Age & 52.1 & 54.6 & 53.3 \\
Mean age (years) & $31-71$ & $41-75$ & $31-75$ \\
Age range (years) & & & \\
\hline
\end{tabular}

Data are $n$ values except where indicated otherwise.

NCM: non-cross-linked native collagen membrane, DCM: dehydrothermally cross-linked collagen membrane.

emergency surgical procedure involving healing around the abutment connection was performed (Figure 2). The remaining 28 patients (mean age, 53.4 years; range, 31 to 75 years) experienced no critical adverse events (Table 1).

The implants were distributed as follows: incisor, $n=10$ (35.7\%); bicuspid, $n=7$ (25\%); and molar, $n=11$ (39.3\%). The 30 implants investigated in these patients comprised the following 4 models, all of which had an internal-connection design: Implantium and NR line ${ }^{\circledR}$ (Dentium), $n=22$ (78.6\%); TS III ${ }^{\circledR}$ (Osstem), $n=2$ (7.1\%); Bone Leve ${ }^{\circledR}$ (Straumann), $n=3$ (10.7\%); and Luna ${ }^{\circledR}$ (Shinhung), $\mathrm{n}=1(3.6 \%)$. The diameter of the most commonly used implant was $4.8 \mathrm{~mm}(\mathrm{n}=8,28.6 \%)$, while the other implants had the following diameters: $3.8 \mathrm{~mm}(\mathrm{n}=8,28.6 \%), 4.3 \mathrm{~mm}(\mathrm{n}=5,17.9 \%), 3.3$ $\mathrm{mm}(\mathrm{n}=2,7.1 \%)$, and $3.6,4.1,4.5,5.0$, and $6.0 \mathrm{~mm}(\mathrm{n}=1$ each, $17.9 \%)$. The implants had the following lengths: $10 \mathrm{~mm}(n=19$, $67.9 \%), 8 \mathrm{~mm}(\mathrm{n}=5,17.9 \%), 12 \mathrm{~mm}(\mathrm{n}=2,7.1 \%)$, and 8.5 and 9 $\mathrm{mm}(\mathrm{n}=1$ for each, 7.1\%, Table 2).

\section{Clinical findings}

In the present study we attempted to determine the clinical feasibility of using DCM compared with NCM for treating human 
Table 2. Characteristics of implants.

\begin{tabular}{|c|c|c|c|}
\hline Characteristic & $\begin{array}{l}\text { NCM group } \\
(\mathrm{n}=14)\end{array}$ & $\begin{array}{l}\text { DCM group } \\
\quad(n=14)\end{array}$ & Total \\
\hline \multicolumn{4}{|l|}{ Implant system } \\
\hline Implantium and NR line, Dentium & 9 & 13 & 22 \\
\hline TS III, Osstem & 1 & 1 & 2 \\
\hline Bone level, Straumann & 3 & 0 & 3 \\
\hline Shinhung, Luna implant system & 1 & 0 & 1 \\
\hline \multicolumn{4}{|l|}{ Location } \\
\hline Incisor region & 7 & 3 & 10 \\
\hline Bicuspid region & 2 & 5 & 7 \\
\hline Molar region & 5 & 6 & 11 \\
\hline \multicolumn{4}{|l|}{ Diameter (mm) } \\
\hline 3.3 & 2 & 0 & 2 \\
\hline 3.6 & 0 & 1 & 1 \\
\hline 3.8 & 3 & 5 & 8 \\
\hline 4.1 & 1 & 0 & 1 \\
\hline 4.3 & 3 & 2 & 5 \\
\hline 4.5 & 0 & 1 & 1 \\
\hline 4.8 & 3 & 5 & 8 \\
\hline 5.0 & 1 & 0 & 1 \\
\hline 6.0 & 1 & 0 & 1 \\
\hline \multicolumn{4}{|l|}{ Length (mm) } \\
\hline 8.0 & 0 & 5 & 5 \\
\hline 8.5 & 1 & 0 & 1 \\
\hline 9.0 & 0 & 1 & 1 \\
\hline 10.0 & 13 & 6 & 19 \\
\hline 12.0 & 0 & 2 & 2 \\
\hline
\end{tabular}

Data are $n$ values except where indicated otherwise.

NCM: non-cross-linked native collagen membrane, DCM: dehydrothermally cross-linked collagen membrane.

peri-implant dehiscence defects. We therefore conducted experiments that excluded bone graft materials from the evaluations, and assessed only the membranes themselves.

Five defect sites (two sites in the NCM group and three sites in the DCM group) showed soft-tissue dehiscence defects and membrane exposure during the early healing period. Despite the presence of plaque accumulation and signs of mild inflammation around the soft-tissue dehiscence defects, additional necrosis and secondary dehiscence defects did not occur. Spontaneous secondary closures were successfully completed, and no significant differences were observed between the two groups at the 8-week follow-up.

The DW value decreased from $3.8 \pm 1.3$ to $0.4 \pm 0.9 \mathrm{~mm}$ in the NCM group and from $3.5 \pm 1.1$ to $1.7 \pm 1.6 \mathrm{~mm}$ in the DCM group; the corresponding $\triangle \mathrm{DW}$ values were $3.5 \pm 1.2$ and $1.7 \pm 2.2 \mathrm{~mm}$, respectively. Similarly, the $\mathrm{DH}$ value decreased from $5.1 \pm 2.4$ to $0.2 \pm 0.6 \mathrm{~mm}$ in the NCM group and from $4.5 \pm 2.2$ to $1.1 \pm 1.2 \mathrm{~mm}$
Table 3. Clinical measurements of horizontal and vertical dehiscence defects.

\begin{tabular}{|lccc|}
\hline & NCM group $(m m, n=14)$ & DCM group $(m m, n=14)$ & $P$-value \\
\hline DW & & & \\
Baseline & $3.8 \pm 1.3$ & $3.5 \pm 1.1$ & 0.444 \\
16 weeks & $0.4 \pm 0.9$ & $1.7 \pm 1.6$ & $0.010^{\text {a) }}$ \\
DH & & & \\
Baseline & $5.1 \pm 2.4$ & $4.5 \pm 2.2$ & 0.436 \\
16 weeks & $0.2 \pm 0.6$ & $1.1 \pm 1.2$ & $0.018^{\text {a) }}$ \\
$\Delta$ DW & $3.5 \pm 1.2$ & $1.7 \pm 2.2$ & $0.016^{\text {a) }}$ \\
$\Delta$ DH & $5.0 \pm 2.5$ & $2.9 \pm 2.3$ & $0.031^{\text {a) }}$ \\
\hline
\end{tabular}

Data are mean $\pm S D$ values.

NCM: non-cross-linked native collagen membrane, DCM: dehydrothermally cross-linked collagen membrane, DW: defect width, DH: defect height, $\triangle \mathrm{DW}$ : DW (re-entry surgery) - DW (baseline), $\triangle \mathrm{DH}$ : DH (re-entry surgery) - DH (baseline).

a) Statistically significant difference in clinical measurements between the two groups $(P<0.05)$.

Table 4. Visual analog scale (VAS) scores assessing the ease of membrane manipulation and maintenance (0: very good, 10 : very poor).

\begin{tabular}{|lccc|}
\hline Assessment & NCM group $(n=14)$ & DCM group $(n=14)$ & $P$-value \\
\hline Manipulation & $0.5 \pm 1.0$ & $1.3 \pm 1.7$ & 0.119 \\
Maintenance & $0.3 \pm 0.7$ & $1.3 \pm 1.1$ & $0.012^{\text {a) }}$ \\
\hline
\end{tabular}

Data are mean $\pm S D$ values.

NCM: non-cross-linked native collagen membrane, DCM: dehydrothermally cross-linked collagen membrane.

${ }^{\text {a) }}$ Statistically significant difference between the two groups $(P<0.05)$.

in the DCM group, with corresponding $\triangle \mathrm{DH}$ values of $5.0 \pm 2.5$ and $2.9 \pm 2.3 \mathrm{~mm}$, respectively. The $\Delta \mathrm{DW}$ and $\Delta \mathrm{DH}$ values were statistically significant between groups $(P=0.016$ and $P=0.031$, respectively; Table 3).

The clinical assessment of the ease of manipulation and maintenance was based on the VAS scores in the two groups. The VAS score for membrane manipulation did not differ significantly between the NCM group ( $0.5 \pm 1.0$; range, 0 to 3$)$ and the DCM group (1.3 \pm 1.7 ; range, 0 to $5 ; P=0.119)$. In contrast, the VAS score for the maintenance of augmented bone substitutes did differ significantly between the two groups: $0.3 \pm 0.7$ (range, 0 to 2 ) in the NCM group and $1.3 \pm 1.1$ (range, 0 to 3 ) in the DCM group ( $P=0.012$, Table 4).

\section{Radiographic findings}

The mean $\mathrm{HT}$ and corresponding $\Delta \mathrm{HT}$ values were comparable in the two groups (Table 5). The HT values decreased and the corresponding $\Delta H T$ values increased by similar amounts in the two groups, and there were no statistically significant differences among any of the $\Delta H T$ values (Table 4).

The bone density of HT1 as assessed at the time of re-entry surgery did not differ significantly between the NCM group (769.5 \pm 2 18.2 $\mathrm{HU}$; range, 421.1 to $1163.7 \mathrm{HU}$ ) and the DCM group (739.6 \pm $275.1 \mathrm{HU}$; range, 280.4 to $1179.4 \mathrm{HU} ; P=0.752$ ). 


\section{Histological findings}

There was insufficient keratinized gingiva around the implant in six patients, and so histological slides from the remaining 10 DCM and 12 NCM specimens were examined by light microscopy. Soft tissue biopsy was performed to confirm the presence of the remaining membrane fragment. Little Newly formed bone was observed around the grafted bone particles, which were dispersed above the bone bed in all groups. No membrane remnants were observed in any samples of the NCM group. In contrast, partially

Table 5. Measurements made in the radiographic analyses.

\begin{tabular}{|lccc|}
\hline & NCM group $(\mathrm{mm}, \mathrm{n}=14)$ & DCM group $(\mathrm{mm}, \mathrm{n}=14)$ & $P$-value \\
\hline HT1 & & & \\
Baseline & $2.3 \pm 0.8$ & $2.6 \pm 0.8$ & 0.375 \\
16 weeks & $1.7 \pm 1.2$ & $1.6 \pm 1.2$ & 0.734 \\
HT2 & & & \\
Baseline & $2.6 \pm 0.7$ & $3.2 \pm 0.9$ & $0.039^{\text {a) }}$ \\
16 weeks & $2.1 \pm 1.1$ & $2.5 \pm 1.4$ & 0.436 \\
HT3 & & & \\
Baseline & $2.7 \pm 0.8$ & $3.9 \pm 1.2$ & $0.004^{\text {a) }}$ \\
16 weeks & $2.4 \pm 1.4$ & $3.0 \pm 1.4$ & 0.329 \\
$\Delta$ HT1 & $0.6 \pm 0.9$ & $1.0 \pm 1.1$ & 0.277 \\
$\Delta H T 2$ & $0.5 \pm 0.7$ & $0.8 \pm 1.1$ & 0.383 \\
$\Delta H T 3$ & $0.3 \pm 0.9$ & $1.0 \pm 1.0$ & 0.080 \\
\hline
\end{tabular}

Data are mean $\pm S D$ values.

HT1, HT2, and HT3: horizontal thicknesses at 1, 2, and $3 \mathrm{~mm}$ below the top of the implant platform, $\triangle H T x$ : HTx (re-entry surgery) - HTx (baseline), NCM: non-cross-linked native collagen membrane, DCM: dehydrothermally cross-linked collagen membrane.

${ }^{\text {a) }}$ Statistically significant difference between the two groups in radiographic measurements $(P<0.05)$. resorbed DCM leaflets exhibiting structural integrity were clearly identified between the gingival connective tissue and bone substitute materials in the DCM group at magnifications of 100- and 200-fold (Figure 3). Inflammatory processes were not considered to be present in either group, and so these histological results indicate that DCM exhibited sufficient biocompatibility.

\section{DISCUSSION}

The aim of this study was to determine the clinical feasibility of using DCM compared with NCM for treating human peri-implant dehiscence defects. Both types of collagen membrane demonstrated significant improvements in all clinical and radiographic parameters, with no signs or symptoms of severe complications during the postoperative experimental period.

The horizontal and vertical dehiscence defects did not differ significantly between the DCM and NCM groups at baseline. Re-entry surgery was performed after 16 weeks, at which time the clinical condition of the defects was considered to be acceptable, thereby confirming the successful occurrence of GBR. Premature exposure of membranes was found in two samples in the NCM group (14.3\%) and three samples in the DCM group (21.4\%). It is generally known that soft-tissue dehiscence defects and exposure of membrane lead to impaired bone healing and significant deterioration of the defect $[23,24]$. However, notable reduction of bone regeneration and impaired wound healing was not observed in the DCM group. This is consistent with the finding of Moses et al. [25] that a cross-linked collagen membrane was advantageous for GBR procedures. The results obtained in the previous and present studies together indicate that the cross-linking method prevents biodegradation of the collagen membrane and promotes soft-tissue healing without any signs of infection during the secondary healing period [25,26].
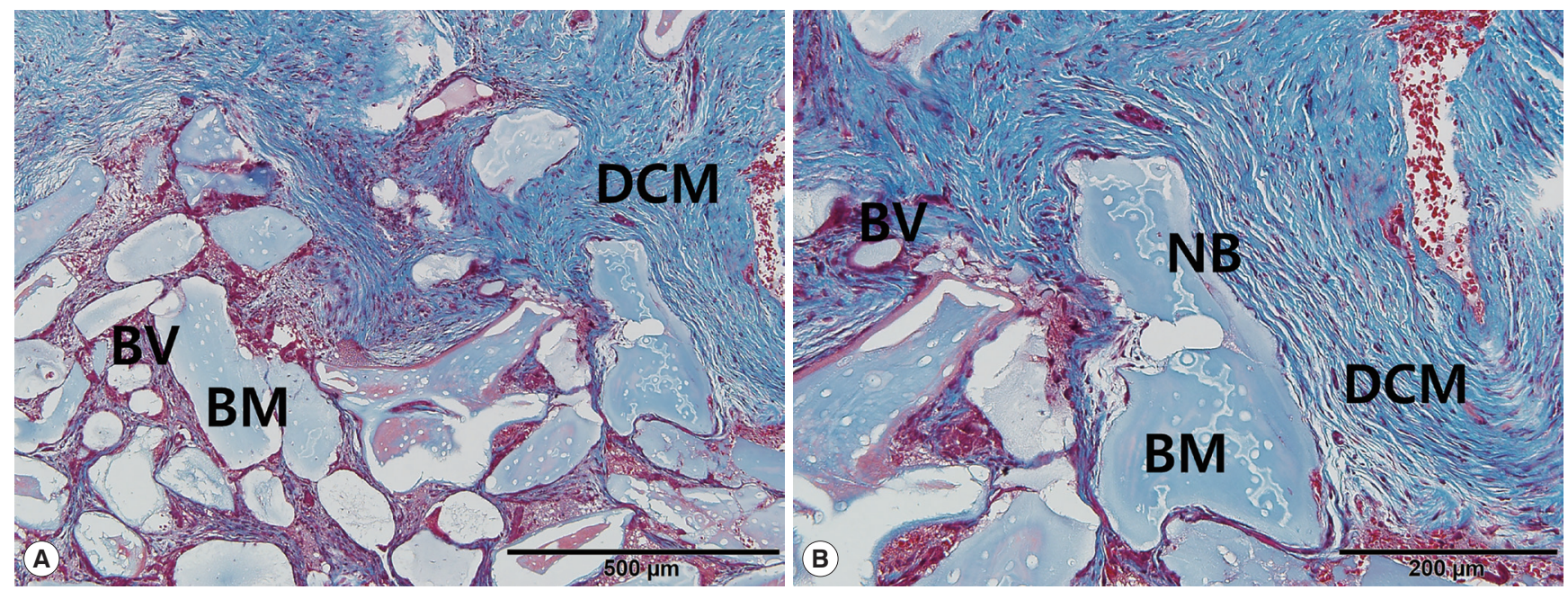

Figure 3. Histological images from the DCM group at the time of re-entry surgery. (A, B) Partially resorbed collagen membrane leaflets exhibiting structural integrity and some blood vessels (BVs) were observed. Bone substitute materials (BM) were surrounded by collagen membranes and BVs. New bone (NB) formation was observed on the BM surface (A: Masson's trichrome stains, $\times 100$, Scale bars, $500 \mu \mathrm{m}$; B: Masson's trichrome stains, $\times 200$, Scale bars, $200 \mu \mathrm{m}$ ). 
The measurements of $\mathrm{HT} 1, \mathrm{HT} 2$, and $\mathrm{HT} 3$ revealed decreases at 16 weeks in both groups compared to baseline, but there were no statistically significant intergroup differences. The bone density of HT1 was measured using CBCT to predict the quality of bone remodeling. The average bone density in both groups was within the general acceptable range (D1 to D3) for bone quality [27]. Regardless of the presence of premature membrane exposure, no significant radiographic or clinical differences were evident.

Histological analyses revealed that the NCM applied at the defect site was significantly absorbed at 4 weeks and almost completely absorbed at 16 weeks [28]. NCM reportedly shows rapid degradation and good biocompatibility, whereas physically cross-linked membrane shows slow degradation but with a similarly low cytotoxicity $[29,30]$. However, it was difficult to verify these findings with the histological results obtained at 16 weeks in the present study. The remaining unabsorbed DCM fragment was observed histologically only in the DCM group, whereas no NCM fragments were observed in the NCM group. This histological result for the DCM group was similar to that for the NCM group and it confirmed the presence of good biocompatibility. However, because this study conducted biopsy sampling in the keratinized gingival tissue zone above the cover screw, the amount of new bone and pattern of bone resorption could not be evaluated, and only some grafted bone particles and integration into new bone were observed.

Depending on the type, structure, degree of cross-linking, and surface-treatment method, it is crucial to determine the mechanical, chemical, and physical properties of the collagen membrane $[11,31]$. The DHT cross-linking technique increases the number of amino-acid side chains between collagen molecules and unwinds the triple-helix structure of collagen [32]. The fibroblast binding sites ( $\alpha 1 \beta 1$ and $\alpha 2 \beta 1$ integrins) are subsequently modified, but it is not yet clear if these physical changes determine the major mechanical and biochemical characteristics of DCM $[9,33]$. Compared to the conventional DHT cross-linking method, which requires 3 to 5 days of dehydration, the collagen membrane used in the present study was dehydrated at $100^{\circ} \mathrm{C}$ under vacuum pressure (1 torr), which reduced the dehydration period to 24 hours. Like the physical cross-linking method using UV radiation, this represents a more effective and rapid cross-linking method [32].

The previous and present studies found that DCM was stiffer and more hydrophobic than DCM, which is attributable to the increased binding between the carboxyl and amino groups of the adjacent collagen molecules $[33,34]$. Despite the constraints of the crosslinking technique, when performing GBR at the time of implant placement it was found that the membrane could be easily manipulated, without any significant differences between the two groups. While there was a statistically significant difference in the clinician's subjective judgement of the maintenance of localized bone particles, there was no clinically meaningful difference in the VAS score between the NCM $(0.3 \pm 0.7)$ and DCM $(1.3 \pm 1.1)$ groups $(P=0.119)$. Therefore, clinicians can use the DHT cross-linking method to control the biodegradation rate of the membrane, allowing the easy and effective use of DCM to achieve successful bone augmentation.

In this study, the different types of implants fixtures had the same SLA surfaces with bone level fixtures; in other words, they had very similar designs and surfaces. A recent systematic review revealed no significant difference between SLA implants types in success rate or marginal bone loss [35]; therefore, although this may pose as a limitation, its effect can be assumed to be minimal. Moreover, the change in bone graft material during the study that was necessitated by our parallel research findings also acts as a limitation. Despite the limitations, the present randomized clinical study indicates that the clinical expediency, biocompatibility, and more enhanced degradation resistance are similar for DCM and NCM. DCM can therefore be used for GBR around serious peri-implant defects.

\section{CONFLICT OF INTEREST}

No potential conflict of interest relevant to this article was reported.

\section{ACKNOWLEDGEMENTS}

This work was supported by the Medical Device Comparative Clinical Trial and Performance Evaluation Program funded by the Small and Medium Business Administration (SMBA, Korea).

\section{ORCID}

Jae-Hong Lee http://orcid.org/0000-0002-2375-0141

Jung-Seok Lee http://orcid.org/0000-0003-1276-5978

Won-Sun Baek http://orcid.org/0000-0002-3427-4101

Hyun-Chang Lim http://orcid.org/0000-0001-7695-1708

Jae-Kook Cha http://orcid.org/0000-0002-6906-7209

Seong-Ho Choi http://orcid.org/0000-0001-6704-6124

Ui-Won Jung http://orcid.org/0000-0001-6371-4172

\section{REFERENCES}

1. Chiapasco M, Zaniboni M. Clinical outcomes of GBR procedures to correct peri-implant dehiscences and fenestrations: a systematic review. Clin Oral Implants Res 2009;20 Suppl 4:113-23.

2. Retzepi M, Donos N. Guided Bone Regeneration: biological principle and therapeutic applications. Clin Oral Implants Res 2010; 21:567-76.

3. Locci $P$, Calvitti M, Belcastro S, Pugliese M, Guerra M, Marinucci L, et al. Phenotype expression of gingival fibroblasts cultured on membranes used in guided tissue regeneration. J Periodontol 1997;68:857-63.

4. Schwarz F, Rothamel D, Herten M, Wüstefeld M, Sager M, Ferrari $D$, et al. Immunohistochemical characterization of guided bone regeneration at a dehiscence-type defect using different barrier 
membranes: an experimental study in dogs. Clin Oral Implants Res 2008;19:402-15.

5. Behring J, Junker R, Walboomers XF, Chessnut B, Jansen JA. Toward guided tissue and bone regeneration: morphology, attachment, proliferation, and migration of cells cultured on collagen barrier membranes. A systematic review. Odontology 2008;96:1-11.

6. Schwarz F, Rothamel D, Herten M, Sager M, Becker J. Angiogenesis pattern of native and cross-linked collagen membranes: an immunohistochemical study in the rat. Clin Oral Implants Res 2006; 17:403-9.

7. Sela MN, Kohavi D, Krausz E, Steinberg D, Rosen G. Enzymatic degradation of collagen-guided tissue regeneration membranes by periodontal bacteria. Clin Oral Implants Res 2003;14:263-8.

8. Jung RE, Fenner $\mathrm{N}$, Hämmerle $\mathrm{CH}$, Zitzmann NU. Long-term outcome of implants placed with guided bone regeneration (GBR) using resorbable and non-resorbable membranes after 12-14 years. Clin Oral Implants Res 2013;24:1065-73.

9. Drexler JW, Powell HM. Dehydrothermal crosslinking of electrospun collagen. Tissue Eng Part C Methods 2011;17:9-17.

10. Schwarz F, Sager M, Rothamel D, Herten M, Sculean A, Becker J. Use of native and cross-linked collagen membranes for guided tissue and bone regeneration. Schweiz Monatsschr Zahnmed 2006; 116:1112-23.

11. Charulatha V, Rajaram A. Influence of different crosslinking treatments on the physical properties of collagen membranes. Biomaterials 2003;24:759-67.

12. Olde Damink LH, Dijkstra PJ, van Luyn MJ, van Wachem PB, Nieuwenhuis $\mathrm{P}$, Feijen J. Cross-linking of dermal sheep collagen using a water-soluble carbodiimide. Biomaterials 1996;17:765-73.

13. Zahedi S, Bozon C, Brunel G. A 2-year clinical evaluation of a diphenylphosphorylazide-cross-linked collagen membrane for the treatment of buccal gingival recession. J Periodontol 1998;69: 975-81.

14. Becker J, Al-Nawas B, Klein MO, Schliephake $H$, Terheyden $H_{\text {, }}$ Schwarz F. Use of a new cross-linked collagen membrane for the treatment of dehiscence-type defects at titanium implants: a prospective, randomized-controlled double-blinded clinical multicenter study. Clin Oral Implants Res 2009;20:742-9.

15. Annen BM, Ramel $\mathrm{CF}$, Hämmerle $\mathrm{CH}$, Jung RE. Use of a new crosslinked collagen membrane for the treatment of peri-implant dehiscence defects: a randomised controlled double-blinded clinical trial. Eur J Oral Implantol 2011;4:87-100.

16. Marzec E, Pietrucha K. The effect of different methods of crosslinking of collagen on its dielectric properties. Biophys Chem 2008:132:89-96.

17. Haugh MG, Jaasma MJ, O'Brien FJ. The effect of dehydrothermal treatment on the mechanical and structural properties of collagen-GAG scaffolds. J Biomed Mater Res A 2009;89:363-9.

18. Rothamel D, Benner M, Fienitz T, Happe A, Kreppel M, Nickenig $\mathrm{HJ}$, et al. Biodegradation pattern and tissue integration of native and cross-linked porcine collagen soft tissue augmentation matrices - an experimental study in the rat. Head Face Med 2014;
10:10.

19. Morris K. Revising the Declaration of Helsinki. Lancet 2013;381: 1889-90.

20. Stoecklin-Wasmer $C$, Rutjes $A W$, da Costa $B R$, Salvi $G E$, Jüni $P$, Sculean A. Absorbable collagen membranes for periodontal regeneration: a systematic review. J Dent Res 2013;92:773-81.

21. Gholami GA, Najafi B, Mashhadiabbas F, Goetz W, Najafi S. Clinical, histologic and histomorphometric evaluation of socket preservation using a synthetic nanocrystalline hydroxyapatite in comparison with a bovine xenograft: a randomized clinical trial. Clin Oral Implants Res 2012;23:1198-204.

22. Faul F, Erdfelder $E_{1}$ Buchner A, Lang AG. Statistical power analyses using $\mathrm{G}^{*}$ Power 3.1: tests for correlation and regression analyses. Behav Res Methods 2009;41:1149-60.

23. Lorenzoni M, Pertl C, Polansky RA, Jakse N, Wegscheider WA. Evaluation of implants placed with barrier membranes. A restrospective follow-up study up to five years. Clin Oral Implants Res 2002;13:274-80.

24. Donos N, Kostopoulos L, Karring T. Alveolar ridge augmentation using a resorbable copolymer membrane and autogenous bone grafts. An experimental study in the rat. Clin Oral Implants Res 2002;13:203-13.

25. Moses 0, Pitaru S, Artzi Z, Nemcovsky CE. Healing of dehiscencetype defects in implants placed together with different barrier membranes: a comparative clinical study. Clin Oral Implants Res 2005; 16:210-9.

26. Friedmann A, Strietzel FP, Maretzki B, Pitaru S, Bernimoulin JP. Observations on a new collagen barrier membrane in 16 consecutively treated patients. Clinical and histological findings. J Periodontol 2001;72: 1616-23.

27. Turkyilmaz I, McGlumphy EA. Influence of bone density on implant stability parameters and implant success: a retrospective clinical study. BMC Oral Health 2008;8:32.

28. Owens KW, Yukna RA. Collagen membrane resorption in dogs: a comparative study. Implant Dent 2001;10:49-58.

29. Rothamel D, Schwarz F, Sculean A, Herten M, Scherbaum W, Becker J. Biocompatibility of various collagen membranes in cultures of human PDL fibroblasts and human osteoblast-like cells. Clin Oral Implants Res 2004;15:443-9.

30. Veríssimo DM, Leitão RF, Ribeiro RA, Figueiró SD, Sombra AS, Góes $\mathrm{JC}$, et al. Polyanionic collagen membranes for guided tissue regeneration: Effect of progressive glutaraldehyde cross-linking on biocompatibility and degradation. Acta Biomater 2010;6:4011-8.

31. Tierney CM, Haugh MG, Liedl J, Mulcahy F, Hayes B, O'Brien FJ. The effects of collagen concentration and crosslink density on the biological, structural and mechanical properties of collagenGAG scaffolds for bone tissue engineering. J Mech Behav Biomed Mater 2009;2:202-9.

32. Weadock KS, Miller EJ, Bellincampi LD, Zawadsky JP, Dunn MG. Physical crosslinking of collagen fibers: comparison of ultraviolet irradiation and dehydrothermal treatment. J Biomed Mater Res 1995;29:1373-9. 
33. Cornwell KG, Lei $P$, Andreadis ST, Pins GD. Crosslinking of discrete self-assembled collagen threads: Effects on mechanical strength and cell-matrix interactions. J Biomed Mater Res A 2007;80:36271.

34. Delgado LM, Bayon Y, Pandit A, Zeugolis DI. To cross-link or not to cross-link? Cross-linking associated foreign body response of collagen-based devices. Tissue Eng Part B Rev 2015;21:298-313.

35. Junker $R$, Dimakis $A$, Thoneick $M$, Jansen JA. Effects of implant surface coatings and composition on bone integration: a systematic review. Clin Oral Implants Res 2009;20 Suppl 4:185-206. 